\title{
Sutura intradérmica no tratamento de ferimento cortocontuso em região periorbitária: relato de caso
}

\author{
Intradermal suture for treatment of blunt wound in the periorbital \\ region: case report \\ Sutura intradérmica para el tratamiento de lesión contusa en región \\ periorbitaria: reporte de caso \\ Gabriel MULINARI-SANTOS ${ }^{1}$ \\ Laura GARCIA ${ }^{2}$ \\ João Paulo BONARDI ${ }^{1}$ \\ Breno dos Reis FERNANDES ${ }^{3}$ \\ Bruno Coelho MENDES ${ }^{3}$ \\ Francisley Ávila SOUZA \\ Idelmo Rangel GARCIA JÚNIOR \\ ${ }^{I}$ Doutorando em Cirurgia e Traumatologia Bucomaxilofacial, Faculdade de Odontologia de Araçatuba, \\ UNESP-Univ. Estadual Paulista, 16015-050 Araçatuba-SP, Brasil \\ ${ }^{2}$ Aluna de Graduação, Faculdade de Odontologia de Araçatuba, UNESP-Univ. Estadual Paulista, 16015-050 Araçatuba-SP, Brasil \\ ${ }^{3}$ Mestrando em Cirurgia e Traumatologia Bucomaxilofacial, Faculdade de Odontologia de Araçatuba, \\ UNESP-Univ. Estadual Paulista, 16015-050 Araçatuba-SP, Brasil \\ ${ }^{4}$ Professor em Cirurgia e Traumatologia Bucomaxilofacial, Faculdade de Odontologia de Araçatuba, \\ UNESP-Univ. Estadual Paulista, 16015-050 Araçatuba-SP, Brasil
}

\begin{abstract}
Resumo
A correta abordagem dos ferimentos em face é crucial para manter preservação da estética e funções. A anatomia deve ser analisada nos traumas faciais, além da adequada realização dos princípios cirúrgicos. A observância dos princípios cirúrgicos contribui para melhor cicatrização dos ferimentos faciais, para evitar cicatrizes e alterações musculares. Este relato de caso, sendo um paciente de 66 anos de idade, sexo masculino, admitido na Santa Casa de Araçatuba, relatando ter sofrido acidente no trabalho por animal. No exame clínico intrabucal, extrabucal e exame de imagem constatou-se um ferimento cortocontuso em região periorbitária acometendo margens supra e infraorbitárias, com funções oculares preservadas e sem sinais sugestivos de fratura óssea. Foi feita anestesia local, exploração da ferida para possível remoção de corpos estranhos, irrigação com soro fisiológico e sutura intradérmica. No pós-operatório observou-se o adequado manejo ferimento com uso de sutura intradérmica para síntese, somado as devidas instruções ao paciente, possibilitou um satisfatório resultado funcional e também estético do ferimento cortocontuso.

Descritores: Traumatismos Faciais; Ferimentos e Lesões; Suturas.
\end{abstract}

\section{Abstract}

The correct approach to face injuries is crucial to maintaining preservation of aesthetics and functions. The anatomy must be analyzed in the facial traumas, in addition to the adequate realization of the surgical principles. The observance of surgical principles contributes to a better healing of facial injuries, to evoke scars and muscular alterations. This case report, being a 66 year old male, admitted to Santa Casa de Araçatuba, reported having suffered an accident at work per animal. In the intra-oral, extraoral and imaging examination, a short-blunt wound was observed in the periorbital region, affecting supra and infraorbital margins, with preserved ocular functions and no signs suggestive of bone fracture. Local anesthesia was performed, wound exploration for possible removal of foreign bodies, irrigation with saline solution and intradermal suture. In the postoperative period, it was observed the appropriate management of the wound with the use of intradermal suture for synthesis, together with the instructions given to the patient, which allowed a satisfactory functional and also aesthetic result of the short-blunt wound.

Descriptors: Facial Injuries; Wounds and Injuries; Sutures.

\section{Resumen}

El correcto enfoque de las lesiones en la cara es crucial para mantener la preservación de la estética y las funciones. La anatomía debe ser analizada en los traumas faciales, además de la adecuada realización de los principios quirúrgicos. La observancia de los principios quirúrgicos contribuye a una mejor cicatrización de las heridas faciales, para eivtar cicatrices y alteraciones musculares. Este relato de caso, siendo un paciente de 66 años de edad, sexo masculino, admitido en la Santa Casa de Araçatuba, relatando haber sufrido accidente en el trabajo por animal. En el examen clínico intrabucal, extrabucal y examen de imagen se constató una lesión contusa en región periorbitaria que acomode márgenes supra e infraorbitales, con funciones oculares preservadas y sin signos sugestivos de fractura ósea. Se realizó anestesia local, explotación de la herida para posible remoción de cuerpos extraños, irrigación con suero fisiológico y sutura intradérmica. En el postoperatorio se observó el adecuado manejo de la lesión con el uso de sutura intradérmica para síntesis, sumando las debidas instrucciones al paciente, posibilitó un satisfactorio resultado funcional y también estético de la lesión contusa.

Descriptores: Traumatismos Faciales; Heridas y Lesiones; Suturas.

\section{INTRODUÇÃO}

Os traumas que atingem a face devem ser conduzidos com muita cautela visto que muitas estruturas estéticas e funcionais podem ser acometidas. A região periorbitária é composta pelo osso frontal em sua porção superior, medialmente pelo osso frontal e maxila, porção inferior pela maxila e lateralmente pelo processo frontozigomático ${ }^{1}$.

A correta abordagem do caso e a tentativa de utilizar mecanismos que no momento da correção da ferida, diminuam o aparecimento de cicatrizes e defeitos musculares que não serão harmônicos com a face do paciente ${ }^{2}$.

A sutura intradérmica comum promove notoriamente uma boa síntese das camadas da pele apenas expondo os bordos do primeiro e último nós de sutura com um excelente resultado estético ${ }^{3}$.

\section{CASO CLÍNICO}

Paciente do sexo masculino, leucoderma, 66 anos de idade, foi admitido na Santa casa de Araçatuba relatando ter sofrido acidente de trabalho no manejo com animal de grande porte. Ao exame clínico o paciente apresentava ferimento cortocontuso na região periorbitária esquerda, acometendo região supra e infraorbital, com aproximadamente $7 \mathrm{~cm}$, as funções oculares estavam preservadas (Figura 1). Foi realizada tomografia computadorizada (Figura 2), sendo a imagem não sugestiva de traços de fratura. Procedeu-se com anestesia local, inspeção da ferida para possível remoção de corpo estranho, e irrigação com soro fisiológico e a realização da sutura intradérmica (Figura 3). No pós-operatório de 21 dias pode-se notar o bom reparo da ferida com satisfatório resultado estético e mínima cicatriz (Figura 4). 


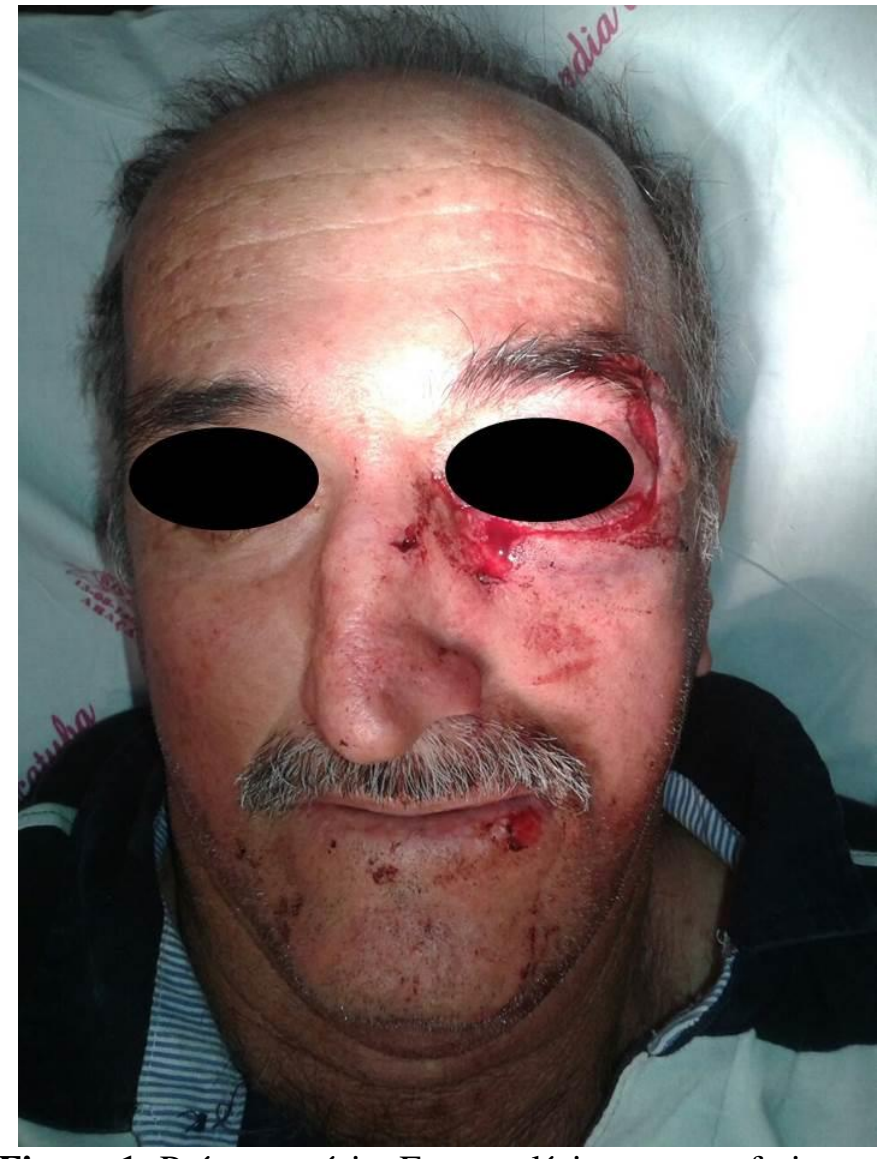

Figura 1: Pré-operatório. Exame clínico nota-se ferimento cortocontuso em margem infraorbitária esquerda.

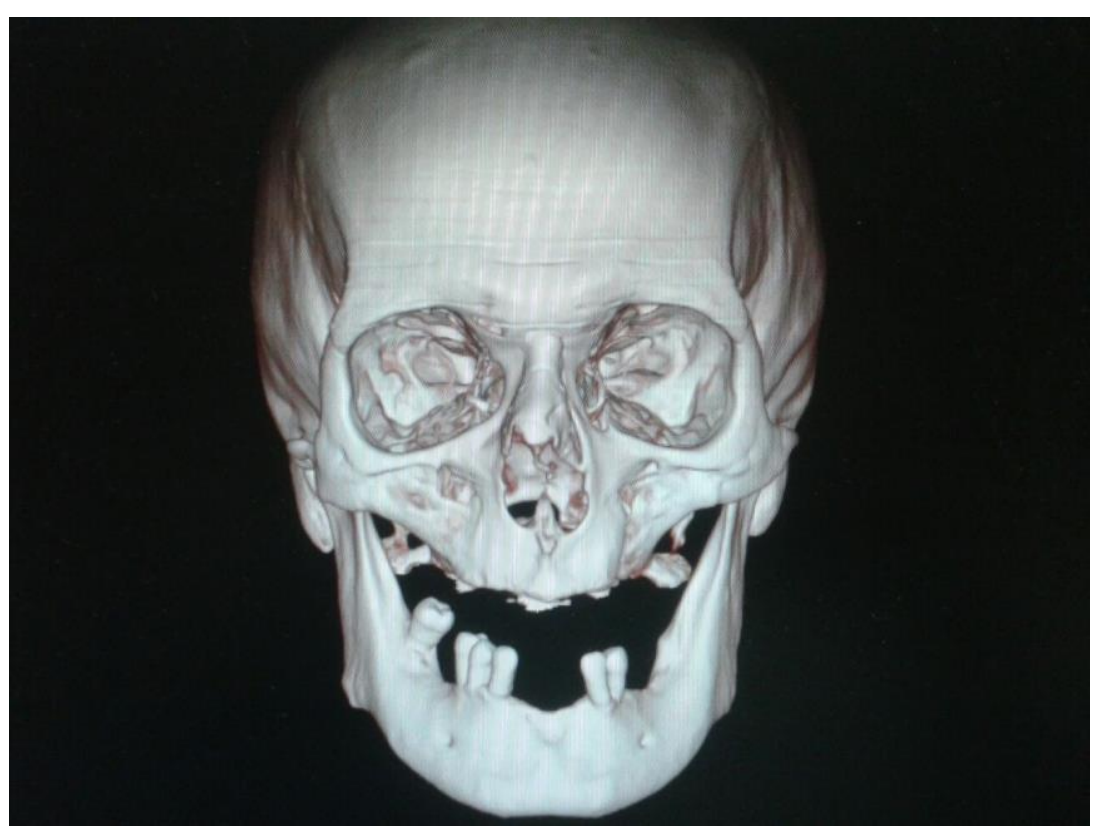

Figura 2: Tomografia Computadorizada em reconstrução tridimensional, revelando paredes ósseas íntegras e sem fraturas.

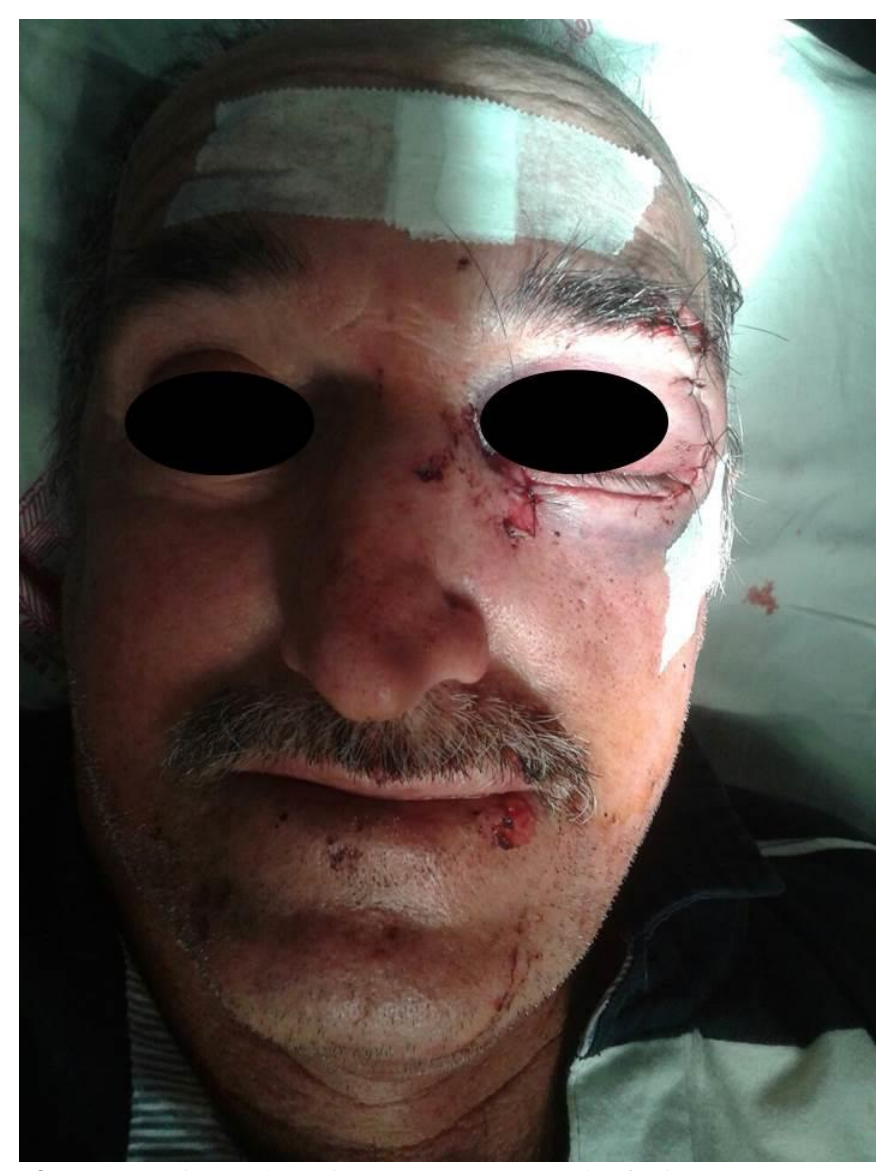

Figura 3: Sutura intradérmica para síntese do ferimento cortocontuso.

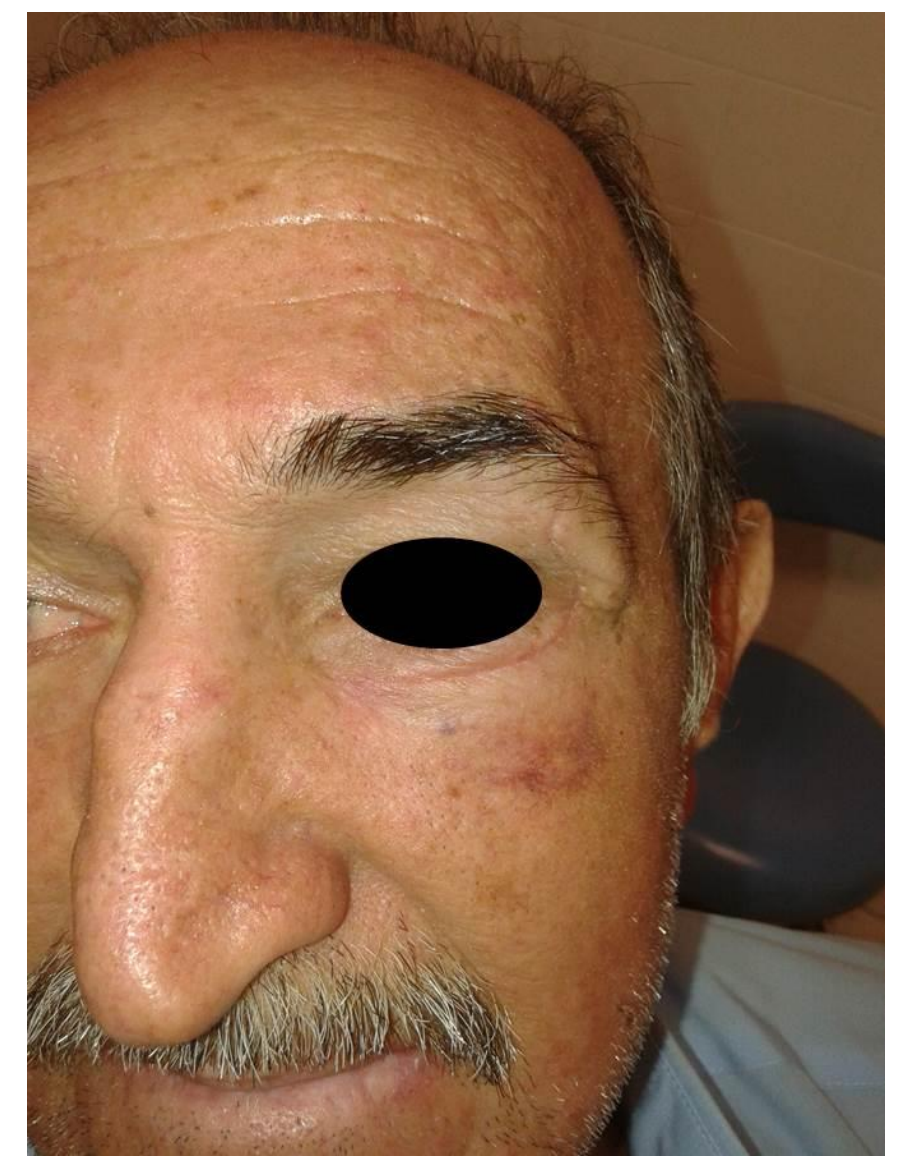

Figura 4: Paciente 21 dias após a cicatrização do tecido, apresentando resultado estético muito satisfatório.

\section{DISCUSSÃO}

A forma como ocorre o manejo da ferida e a conduta que o profissional irá adotar tem um influência significativa no resultado final da cicatrização das áreas afetadas ${ }^{3}$. Desde a habilidade do profissional, até a escolha do tipo da sutura, levando em consideração as características anatômicas em que ocorreu a injúria, a estética e a adequada coaptação das bordas do tecido auxiliam na cicatrização por primeira intenção ${ }^{5}$.

A sutura intradérmica consiste em uma sutura estética ${ }^{6}$ É realizada introduzindo na derme, a agulha paralela ao ângulo de uma extremidade da lesão, amarrando o nó, que não será exposto na superfície. Depois disso, prossegue de maneira idêntica à exposta anteriormente, até atingir a outra extremidade. Para terminá-lo, a agulha deve passar perpendicular ao ângulo de abertura deste fim, ou seja, contendo toda e apenas a derme ${ }^{4}$.

Uma característica crucial para um bom resultado estético é seguir as linhas de força de Kraissl. Estas linhas correspondem às áreas de menor tensão da superfície corporal, quando da realização de incisões e suturas da pele, sendo a sutura intradérmica, uma técnica que minimiza a tensão demasiada nas margens da ferida e consegue mantê-la hermeticamente selada, obtendo bordas mais lineares ${ }^{7}$.

Comparando-se a sutura intradérmica com a sutura simples, com o decorrer da cicatrização fica evidente que o reparo da sutura intradérmica, por não necessitar de pontos externos, garante o melhor resultado estético ${ }^{8}$.

No caso apresentado foi realizada a sutura intradérmica pela demanda estética da área da injúria, tornando mais fácil para o paciente se reintroduzir em seu meio social após o acidente.

\section{CONCLUSÃO}

O emprego da técnica cirúrgica adequada implica na indicação correta da sutura mais adequada à necessidade de cada caso individualmente. A sutura intradérmica, ao encerrar a ferida cutânea sob baixa tensão obtém cicatrizes de boa qualidade, com resultados satisfatórios para áreas estéticas como a região periorbitária.

\section{REFERÊNCIAS}

1. Zide BM, Jelks G. Surgical anatomy of orbital. New York: Raven Press; 1985. 
2. Gomes OM, Amaral ASC, Gonçalves AJV, Brito ASP, Monteiro ELC. New suture techniques for best esthetic skin healing. Acta Cir Bras. 2012; 27(7):505-08.

3. Tessier P. Complications of facial trauma: principles of late reconstruction. Ann Plast Surg. 1986; 17(5):411-20.

4. Rowe NLC, Williams, JL. Maxilofacial injuries. New York: Churchill Livingstone; 1985, p. 435-538.

5. Peterson LJ, Ellis E, Hupp JR. Cirurgia Oral e Maxilofacial Contemporânea. 3.ed. Rio de Janeiro: Guanabara Koogan; 2000. p. 487-511.

6. Vetter JD, Topazian RG, Goldeberg MH, Smith DG Facial fractures occuring in a medium-sized metropolitan area: recent trends. Int J Oral Maxillofac Surg. 1991; 20(4):214-16

7. Manganello LCS, Soares CR, Santos LCB. Fraturas do malar: diagnóstico e tratamento. Rev Ass Paul Cirur Dent. 1982; 36:100

8. Dingman RO, Natvig P. Cirurgia das fraturas faciais. 3.ed. São Paulo: Santos; 2004

\section{CONFLITO DE INTERESSES}

Os autores declaram não haver conflitos de interesse.

\section{AUTOR PARA CORRESPONDÊNCIA}

Gabriel Mulinari dos Santos

gabriel_mulinari@hotmail.com

Submetido em 30/03/2018

Aceito em 30/05/2018 